\title{
FAST PORTRAIT SEGMENTATION WITH HIGHLY LIGHT-WEIGHT NETWORK
}

\author{
Yuezun $\mathrm{Li}^{1 *}$, Ao Luo ${ }^{2 *}$ and Siwei Lyu ${ }^{1}$ \\ ${ }^{1}$ University at Albany, State University of New York, USA \\ ${ }^{2}$ University of Electronic Science and Technology of China
}

\begin{abstract}
In this paper, we describe a fast and light-weight portrait segmentation method based on a new highly light-weight backbone (HLB) architecture. The core element of HLB is a bottleneck-based factorized block (BFB) that has much fewer parameters than existing alternatives while keeping good learning capacity. Consequently, the HLB-based portrait segmentation method can run faster than the existing methods yet retaining the competitive accuracy performance with state-of-the-arts. Experiments conducted on two benchmark datasets demonstrate the effectiveness and efficiency of our method.
\end{abstract}

Index Terms - Portrait segmentation, HLB architecture

\section{INTRODUCTION}

The proliferation of smart phones and social portals (e.g., Facebook, Twitter) has made capturing and sharing personal photos ever more convenient. Many mobile photo editing software is based on the function of portrait segmentation, which is the task to separate the region corresponding to the subject's head and upper-body from a selfie photo for subsequent operations such as background replacement, hair style change, and non-photo realistic rendering of the portrait.

Currently, the most effective portrait segmentation methods are based on convolutional neural networks (CNN), e.g., $[1,2,3]$. These methods adopt a general architecture composed of an encoder (backbone) and a decoder network, see Fig.1(a). To achieve good performance, these methods usually employ powerful CNN models as VGG [4] and ResNet [5]. However, such full-blown CNN models may not scale up well in running efficiency when performing portrait segmentation over large number of images and/or on a lean computing platform such as cellphones. For such tasks, we need a fast, light-weight yet powerful portrait segmentation method. Several more recent works, e.g., [6,7], use existing general purpose light-weight network structures such as the MobileNet [8] to improve the overall running efficiency.

In this paper, we describe a fast and light-weight portrait segmentation method based on a new highly light-weight backbone (HLB) architecture. The core element of this backbone architecture is a novel residual block, which we term as

\footnotetext{
* indicates equal contribution.
}

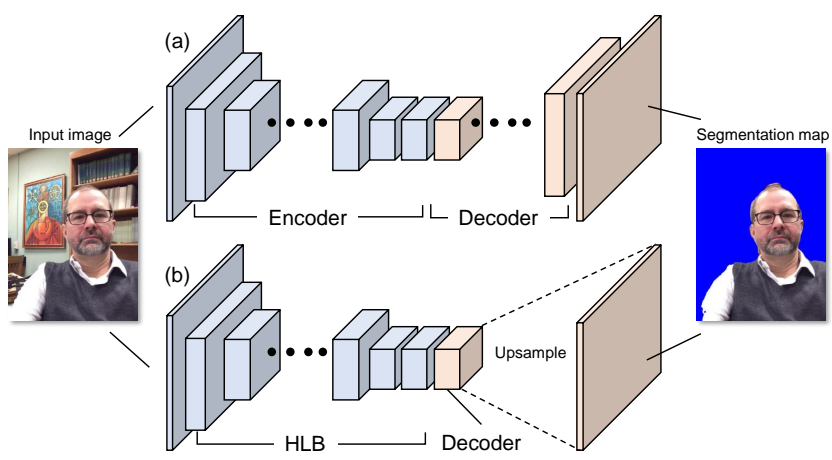

Fig. 1. (a) is the general architecture of portrait segmentation. Given an input image, the encoder extracts the features, which are sent to decoder for segmentation map prediction. (b) is HLB-based portrait segmentation, where the decoder only contains one convolutional layer.

the bottleneck-based factorized block (BFB), combining the bottleneck [5] and factorized layers [9]. The bottleneck structure is composed by two $1 \times 1$ convolutional layers at the beginning and the ending of block, which reduces the width of feature maps inside the block. The factorized layer replaces the standard 2D convolution kernels with two 1D convolution kernels to reduce the number of parameters. The existing methods usually employ multiple layers as decoder to generate the favorable results. In contrast, due to the good learning capacity of HLB architecture, we can achieve the competitive results by only using a single convolutional layer as the decoder (Fig.1(b)). Experiments conducted on two benchmark datasets demonstrate the effectiveness and efficiency of our method.

\section{HLB ARCHITECTURE}

Fig.2 shows the overall structure of the HLB architecture for portrait segmentation. It takes input image of $512 \times 512$ pixels and output the mask of segmented portrait.

The overall structure of HLB architecture is similar to that of [10], which starts with two downsampler blocks (DSB) that downsample the feature map by concatenating the output of a $3 \times 3$ convoluational layer with stride 2 and a max-pooling. It is followed by five bottleneck-based factorized blocks (BFB). After that, another DSB block is used to further downsample the feature map, which is then followed by another set of eight BFBs. 


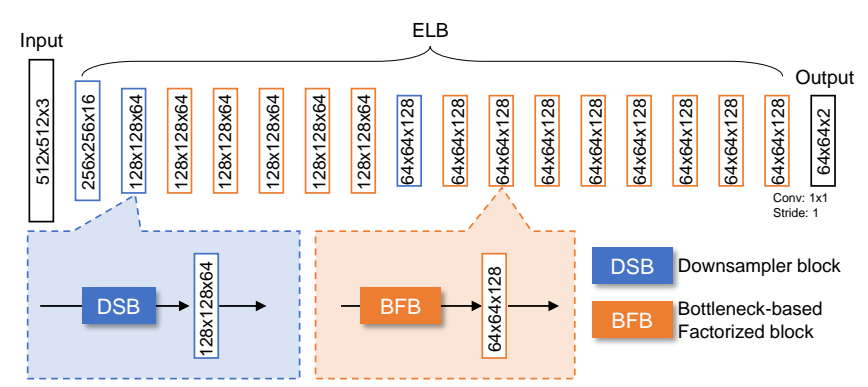

Fig. 2. Overview of HLB-based portrait segmentation. The volumes are feature maps generated by layers. Specifically, the blue and orange feature map are generated by downsampler block (DSB) and bottleneck-based factorized block (BFB) respectively. The decoder is a single $1 \times 1$ convolution layer which converts the last feature map to a segmentation map.

\subsection{Bottleneck-based Factorized Blocks}

The bottleneck-based factorized block (BFB) is the core element of the HLB architecture, which is a special type of residual block [5].

Residual block. A residual block can be defined as

$$
\mathbf{y}=\mathcal{F}\left(\mathbf{x},\left\{W_{i}\right\}\right)+\mathbf{x},
$$

where $\mathbf{x}$ and $\mathbf{y}$ are the input and output feature map, and $\mathcal{F}\left(\mathbf{x},\left\{W_{i}\right\}\right)$ is the residual mapping function implemented as stacked non-linear layers. There are two different instances of residual block proposed in original work: bottleneck block, which is composed by a bottleneck structure and one $3 \times 3$ convolutional layers (Fig.3(a)) and non-bottleneck block, which is composed by two $3 \times 3$ convoluational layers (Fig.3(b)). As in [5], the channel number of input feature map in bottleneck block is four times larger than that in the non-bottleneck block.

Specifically, the bottleneck structure consists of two $1 \times 1$ convolutional layers added at the beginning and the ending of the block respectively. The beginning convoluational layer is implemented by a kernel with size of $1 \times 1 \times c_{0} \times c_{1}$, where $c_{0}$ and $c_{1}$ are the number of channels for the input and output feature map, respectively. By setting $c_{1}<c_{0}$, e.g., $c_{1}=c_{0} / 4$, this layer reduces the width of feature maps in block. The ending convolutional layer is implemented by another kernel with size of $1 \times 1 \times c_{1} \times c_{0}$, which aims to recover the width of feature map back to the original dimension $c_{0}$. In other words, the size of feature maps inside block is reduced, which forms a bottleneck for the data flow through it. Therefore, despite the channel number of input feature map in bottleneck block is larger than it in non-bottleneck block, they can have similar number of parameters. In terms of performance, the non-bottleneck block usually achieve slightly more accuracy compared to bottleneck block [10].

BFB. The BFB combines the bottleneck and non-bottleneck blocks together to reduce the number of parameters while retain the similar accuracy. Specifically in our case, we set $c_{1}=c_{0} / 2$ instead of $c_{1}=c_{0} / 4$ for balancing both accuracy

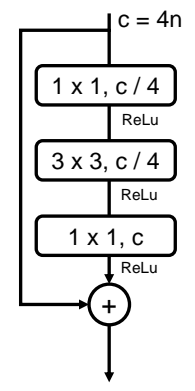

(a) Bottleneck block

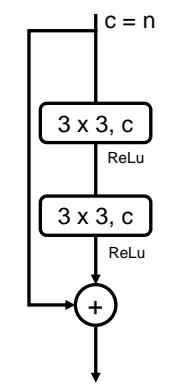

(b) Non-bottleneck block

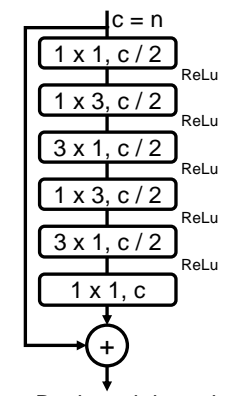

(c) Bottleneck-based factorized block
Fig. 3. Illustration of two residual blocks originated in [5]: Bottleneck block (a) and Non-bottleneck block (b), and our proposed Bottleneck-based factorized block (c). " $c$ " is the channel number of input feature map. " $n$ " is a constant value for better illustrating the difference between each block. Each box inside the block denotes convolution with kernel size and channel number of output feature map.

and efficiency. To further reduce the computation cost, we incorporate the factorized layers [9] into the residual block. Factorized layers are the decomposition of a standard 2D convolutional layer. Specifically, a 2D convolution layer can be approximately viewed as a linear combination of 1D filters. Let $\mathbf{K} \in \mathbb{R}^{h \times w}$ denote the 2D convolutional kernel ${ }^{1}$, where $h$ and $w$ are the height and width of this kernel (usually $h=w$ ). The 2D kernel $\mathbf{K}$ can be decomposed into two 1D convolutional kernels as $\mathbf{k}^{0} \in \mathbb{R}^{1 \times w}, \mathbf{k}^{1} \in \mathbb{R}^{h \times 1}$. Let $\mathbf{u}$ and $\mathbf{v}$ denote the input and output feature map, $*$ denote the convolution operation. The $2 \mathrm{D}$ convolution can be decomposed as

$$
\mathbf{v}=\mathbf{K} * \mathbf{u} \rightarrow \mathbf{k}^{1} *\left(\mathbf{k}^{0} * \mathbf{u}\right) .
$$

As shown in Fig.3(c), we factorize the $3 \times 3$ 2D convolutional kernel into two 1D convolutional kernels with size $1 \times 3$ and $3 \times 1$ respectively. This decomposition further reduces the number of parameters in a standard $3 \times 32 \mathrm{D}$ convolution. Therefore, the new residual block, which we term as bottleneck-based factorized blocks (BFB) can achieve top performance and meanwhile allowing much better efficiency to satisfy the resource limitation of mobile devices.

\subsection{Training}

For portrait segmentation, it is important to generate accurate boundary in favor of the applications such as background replacement. To this end, we propose an adaptive weight loss to increase the penalty of the boundary area. Formally, let $\mathbf{m}_{i}^{p}$ denote the output of class $p$ at $i$-th pixel of predicted segmentation map and $\hat{p}$ denote the correct class of $i$-th pixel. Let $w_{i}$ denote the weight of $i$-th pixel. $N$ is the class number $(N=2$ in our task, portrait region or not). $M$ is the number of pixels in an image. The loss function $L$ can be written as

$$
L=-\frac{1}{M} \sum_{i=0}^{M} w_{i} \log \left(\frac{\exp \left(\mathbf{m}_{i}^{\hat{p}}\right)}{\sum_{p=0}^{N} \exp \left(\mathbf{m}_{i}^{p}\right)}\right) .
$$

\footnotetext{
${ }^{1}$ We omit the channel number of input and output feature map for simplicity.
} 


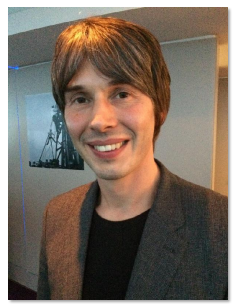

(a)

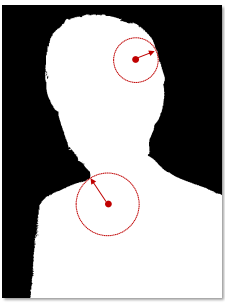

(b)

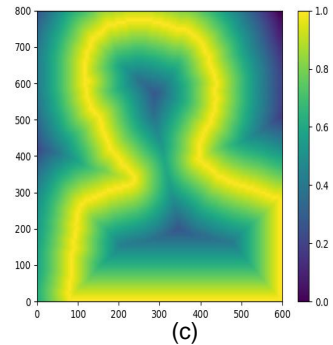

(c)
Fig. 4. Example of an image (a), weight calculation (b) and corresponding boundary weight map (c). In (b), red point denotes pixel and red circle denote searching range.

In general settings [1], $w_{i}$ is equal to 1 , which does not highlight the importance of portrait boundary. In our case, we increase the weight of pixels around boundary. Specifically, we first extract the portrait boundary based on the given mask and calculate the closest distance from each pixel to boundary (Fig.4(b)). Then we create a boundary weight map by normalizing the distance of each pixel to $[0,1]$ (Fig.4(c)). Let $g_{i}$ denote the boundary weight of $i$-th pixel in distance map. The $w_{i}$ is updated to $w_{i}=1+g_{i}$.

\section{EXPERIMENTS}

\subsection{Datasets}

PFCN. The original PFCN dataset contains 1,800 portrait images links from Flicker, where 1,500 images for training and 300 images for testing. The size of these images is $800 \times$ 600 and only one subject is in each image. However, many image links have been expired to date. Only 1,590 images can be downloaded, which includes 1,277 images for training and 263 images for testing. Several examples are shown in Fig.5.

Due to small scale of this dataset, the methods validated on this dataset may not be well generalized to the real-world application. As such, we create another large scale portrait segmentation dataset (PSEG) as complementary.

PSEG. We create a new dataset based on Multi-Human Parsing (MHP) dataset [11], which provides pixel-wise label for semantically consistent regions of body parts. Based on the annotations of upper body (head and shoulder), the portrait image is cropped around the head region with a randomly bounding box, which can cover the entire head and part of shoulders. In this way, we create 20,514 portrait images including 18, 462 images for training and 2, 052 images for testing. In contrast to PFCN dataset, which only contains a single subject in view, PSEG dataset contains at least one subject in an image. Several examples are shown in Fig.5.

\subsection{Experimental Settings}

Implementations. To gather more context in HLB architecture, we use dilated convolutions [14] in the second pair of $1 \times 3$ and $3 \times 1$ convolutional layers for the last eight BFBs, with dilated strides of $1,2,3,4,5,9,13,17$ respectively. We

\begin{tabular}{|c|c|c|c|c|c|}
\hline Methods & $\begin{array}{c}\text { Input size } \\
(\mathrm{w} \times \mathrm{h})\end{array}$ & $\begin{array}{c}\text { FLOPS } \\
(\mathrm{G})\end{array}$ & $\begin{array}{c}\text { Param. } \\
(\mathrm{M})\end{array}$ & FPS & $\begin{array}{c}\mathrm{mIoU} \\
(\%)\end{array}$ \\
\hline Graph-cut [12] & $600 \times 800$ & - & - & - & $80.0^{*}$ \\
\hline PFCN+[1] & $600 \times 800$ & - & - & - & $95.9^{*}$ \\
\hline PDeepLabv2 [1] & $600 \times 800$ & - & - & - & $96.1^{*}$ \\
\hline BSN [3] & $400 \times 400$ & - & - & - & $96.7^{*}$ \\
\hline FCN [13] & $600 \times 800$ & - & - & - & $73.1^{*}$ \\
\hline \hline PIS [7] & $600 \times 800$ & - & - & 23.8 & $95.4^{*}$ \\
\hline PortraitNet [6] & $224 \times 224$ & 0.5 & 2.1 & 203.2 & $96.6^{*}$ \\
\hline \multirow{2}{*}{ HLB (ours) } & $224 \times 224$ & 0.7 & \multirow{2}{*}{0.6} & 714.3 & 94.6 \\
\cline { 2 - 6 } & $512 \times 512$ & 3.8 & & 256.4 & 94.9 \\
\hline
\end{tabular}

Table 1. Quantitative comparison of our method against other existing methods on PFCN dataset. "*” denotes the existing methods are trained and validated on original PFCN dataset which can not be accessed now. See text for details.

implement our method using PyTorch 1.0 framework [15] with CUDA 9.0 on a NIVIDIA 1080ti GPU. The model is trained using Adam optimizer [16] with momentum 0.9 and weight decay $1 e^{-4}$. We use batch size of 16 and set the starting learning rate to $5 e^{-4}$, which is decayed by 0.9 each epoch. The total epoch number is set to 100 .

Data augmentation. To increase the diversity of training data, we horizontally flip the input image with probability 0.5 and augment the color by adjusting contrast and brightness with a factor in $[0.8,1.2]$.

Evaluation metric. The performance is measured using Mean Intersection-over-Union (mIoU) metric, which is defined as $\frac{1}{N} \sum_{0}^{N} \frac{T P}{T P+F P+F N}$, where $N$ is the class number, TP, FP and FN are number of true positives, false positives and false negatives respectively.

\subsection{Comparisons}

We first compare HLB-based portrait segmentation with existing portrait segmentation methods: Graph-cut [12], PFCN+ [1], PDeepLabv2 [1], BSN [3], PIS [7], FCN [13] and PortraitNet [6]. For simplicity, we directly use HLB to denote the HLB-based portrait segmentation. Graph-cut is a graph based method that can extract foreground with user guidance. PFCN+ is adapted from FCN [13] trained on PFCN dataset. PDeepLabv2 and BSN are both built on DeepLabv2 ResNet101 model for portrait segmentation. FCN method denotes directly using the model trained on semantic segmentation for portrait segmentation using 'person' class. PIS and PortraitNet both adopt MobileNetv2 as the backbone architecture for light weight design. Note that all of these existing methods are trained and validated on original PFCN dataset, which can not be accessed now (see Sec.3.1). However, the proposed HLB can only be trained on the current dataset, which is only a subset of original one. Since the code implementations of these existing methods are not realised, we can hardly re-train these methods on the current PFCN dataset. Thus it is difficult to fairly compare HLB with these existing methods. Table 1 shows the performance of each existing method on original PFCN dataset and HLB on current dataset 

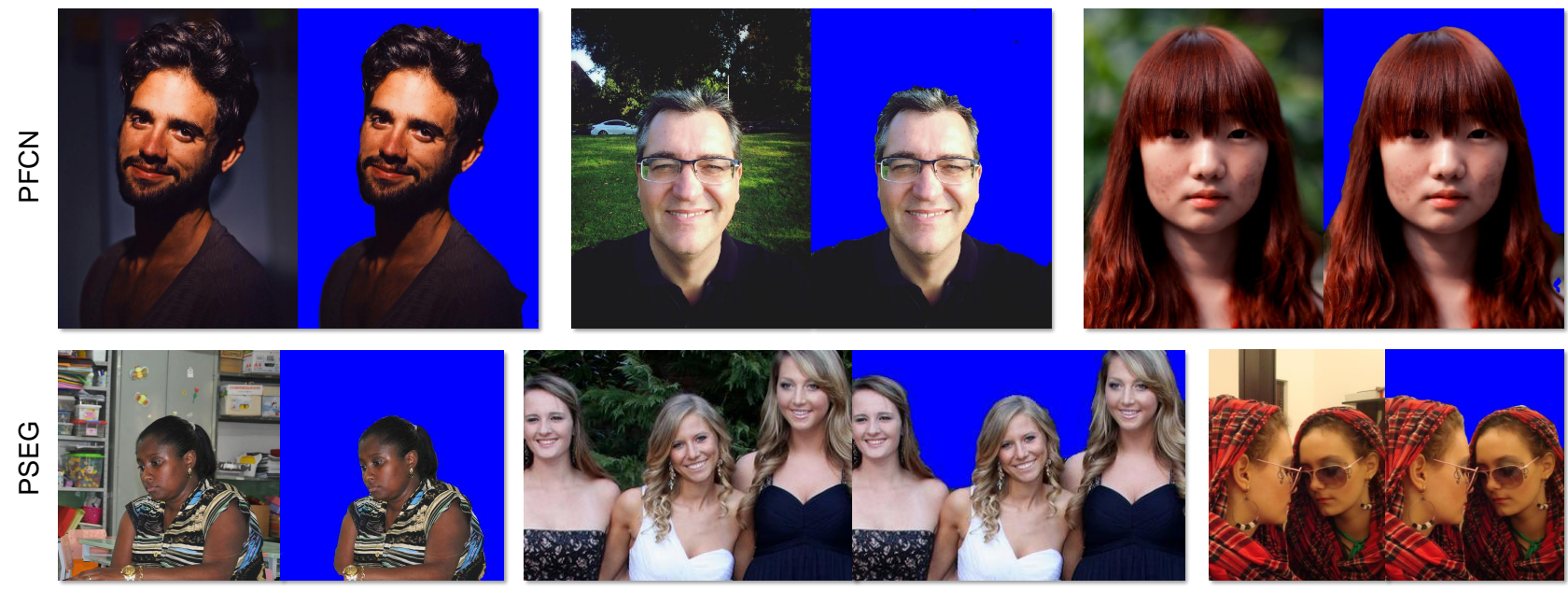

Fig. 5. Example images from PFCN (top) and PSEG (bottom) with corresponding segmentation results using our method.

\begin{tabular}{|c|c|c|c|c|c|}
\hline \multirow{2}{*}{ Methods } & \multirow{2}{*}{ FLOPS (G) } & \multirow{2}{*}{ Param. (M) } & \multirow{2}{*}{ FPS } & \multicolumn{2}{|c|}{ mIoU (\%) } \\
\cline { 5 - 6 } & & & & PFCN & PSEG \\
\hline ERFNet [10] & 13.4 & 2.1 & 128.2 & 94.6 & 93.9 \\
\hline ENet [17] & 2.1 & 0.4 & 220.7 & 94.2 & 93.9 \\
\hline ICNet [18] & 3.5 & 7.7 & 83.3 & 87.8 & 90.0 \\
\hline BiSeNet [19] & 14.5 & 12.8 & 164.0 & 94.5 & 94.4 \\
\hline \hline HLB (ours) & 3.8 & 0.6 & 256.4 & 94.9 & 94.0 \\
\hline
\end{tabular}

\begin{tabular}{|c|c|c|c|c|}
\hline Method & FLOPS (G) & Param. (M) & FPS & mIoU (\%) \\
\hline BFB-DR4 & 1.4 & 0.2 & 370.4 & 93.7 \\
\hline BFB-DR2 & 3.8 & 0.6 & 256.4 & 94.9 \\
\hline
\end{tabular}

Table 3. Comparisons of bottleneck-based factorized blocks with different channel decreasing rate. "DR4" and "DR2" indicate the channel decreasing rate is 4 and 2 respectively.

Table 2. Comparisons of our method with other efficient networks used in semantic segmentation.

2. "FLOPS" denotes the floating-point operations per second. "Param." stands for the number of parameters. Despite the dataset used for evaluation is not exactly same, we highlight that the accuracy performance of proposed HLB is on par with the state-of-the-arts, yet achieves much better running efficiency.

For the model efficiency analysis, only PIS and PortraitNet are built based on light weight architecture. They test the inference time on the same type of GPU (NVIDIA 1080ti) with us, which can achieve 23.8 FPS on input size $600 \times 800$ and 203.2 FPS on input size $224 \times 224$ respectively. Compared to these two methods, the HLB-based portrait segmentation has fewer parameters and runs faster, which achieves 714.3 FPS and 256.4 FPS on input size $224 \times 224$ and $512 \times$ 512 respectively.

Furthermore, we compare HLB with existing efficient networks used in semantic segmentation: ERFNet [10], ENet [17], ICNet [18] and BiSeNet [19]. The backbone of BiSeNet is ResNet18. We re-train these networks on PFCN and PSEG datasets respectively using their default training settings on the same environment as ours. The input image for each method is set to $512 \times 512$, the same as ours. The accuracy and efficiency analysis is reported in Table 2. The results reveal that our HLB-based portrait segmentation method can run 256.4 FPS, which is faster than other methods. Note the ENet has less FLOPS and Parameters than ours, yet it is slower than our method. This is probably due to the ENet has much more skip connections than ours, which is slower

\footnotetext{
${ }^{2}$ The performance of Graph-cut, PFCN+, PDeepLabv2 are referred from [3], FCN is referred from [7].
}

under the implementation of PyTorch. At the same time, our method can achieve the best accuracy performance $(94.9 \%)$ on current PFCN dataset and competitive results (94.0\%) on PSEG dataset. Fig.5 shows several segmentation resutls of our method on two datasets.

\subsection{Ablation Study}

We investigate the effect of the channel decreasing rate in bottleneck of BFB. In this experiment, we enlarge the channel decreasing rate from 2 to 4 . In this way, the width of block will be further reduced. Table 3 shows the quantitative comparison of BFB with channel decreasing rate 4 and 2 on PFCN dataset. From the results we can see by changing the channel decreasing rate to 4 , the FLOPS, number of parameters are notably reduced, which lead to an improvement in FPS from 256.4 to 370.4 . However, the accuracy is degraded notably by $1.2 \%$. Considering the trade-off between accuracy and efficiency, we employ the channel decreasing rate 2 in our setting.

\section{CONCLUSION}

In this paper, we propose an highly light-weight backbone (HLB) architecture for portrait segmentation. The proposed architecture runs much faster than existing methods, yet achieves competitive performance on several datasets. The core element of HLB is a novel bottleneck-based factorized block (BFB), which is the combination of bottleneck structure with factorized layers that can greatly reduce the number of parameters while retaining the good learning capacity. The experiments performed on PFCN and PSEG dataset demonstrates the effectiveness of HLB architecture on portrait segmentation task. 


\section{REFERENCES}

[1] Xiaoyong Shen, Aaron Hertzmann, Jiaya Jia, Sylvain Paris, Brian Price, Eli Shechtman, and Ian Sachs, "Automatic portrait segmentation for image stylization," in Computer Graphics Forum, 2016.

[2] Xiaoyong Shen, Hongyun Gao, Xin Tao, Chao Zhou, and Jiaya Jia, "High-quality correspondence and segmentation estimation for dual-lens smart-phone portraits," in ICCV, 2017.

[3] Xianzhi Du, Xiaolong Wang, Dawei Li, Jingwen Zhu, Serafettin Tasci, Cameron Upright, Stephen Walsh, and Larry Davis, "Boundary-sensitive network for portrait segmentation," in IEEE International Conference on Automatic Face \& Gesture Recognition (FG), 2019.

[4] Karen Simonyan and Andrew Zisserman, "Very deep convolutional networks for large-scale image recognition," arXiv preprint arXiv:1409.1556, 2014.

[5] Kaiming He, Xiangyu Zhang, Shaoqing Ren, and Jian Sun, "Deep residual learning for image recognition," in CVPR, 2016.

[6] Song-Hai Zhang, Xin Dong, Hui Li, Ruilong Li, and Yong-Liang Yang, "Portraitnet: Real-time portrait segmentation network for mobile device," Computers \& Graphics, 2019.

[7] Lingyu Zhu, Tinghuai Wang, Emre Aksu, and JoniKristian Kamarainen, "Portrait instance segmentation for mobile devices," in IEEE International Conference on Multimedia and Expo (ICME), 2019.

[8] Andrew G Howard, Menglong Zhu, Bo Chen, Dmitry Kalenichenko, Weijun Wang, Tobias Weyand, Marco Andreetto, and Hartwig Adam, "Mobilenets: Efficient convolutional neural networks for mobile vision applications," arXiv preprint arXiv:1704.04861, 2017.

[9] Jose Alvarez and Lars Petersson, "Decomposeme: Simplifying convnets for end-to-end learning," arXiv preprint arXiv:1606.05426, 2016.

[10] Eduardo Romera, José M Alvarez, Luis M Bergasa, and Roberto Arroyo, "Erfnet: Efficient residual factorized convnet for real-time semantic segmentation," IEEE Transactions on Intelligent Transportation Systems (TITS), 2017.

[11] Jian Zhao, Jianshu Li, Yu Cheng, Terence Sim, Shuicheng Yan, and Jiashi Feng, "Understanding humans in crowded scenes: Deep nested adversarial learning and a new benchmark for multi-human parsing," in ACM international conference on Multimedia, 2018.
[12] Jianbo Shi and Jitendra Malik, "Normalized cuts and image segmentation," Departmental Papers (CIS), 2000.

[13] Jonathan Long, Evan Shelhamer, and Trevor Darrell, "Fully convolutional networks for semantic segmentation," in CVPR, 2015.

[14] Fisher Yu and Vladlen Koltun, "Multi-scale context aggregation by dilated convolutions," arXiv preprint arXiv:1511.07122, 2015.

[15] Adam Paszke, Sam Gross, Soumith Chintala, Gregory Chanan, Edward Yang, Zachary DeVito, Zeming Lin, Alban Desmaison, Luca Antiga, and Adam Lerer, "Automatic differentiation in pytorch," 2017.

[16] Diederik P Kingma and Jimmy Ba, "Adam: A method for stochastic optimization," arXiv preprint arXiv:1412.6980, 2014.

[17] Adam Paszke, Abhishek Chaurasia, Sangpil Kim, and Eugenio Culurciello, "Enet: A deep neural network architecture for real-time semantic segmentation," arXiv preprint arXiv:1606.02147, 2016.

[18] Hengshuang Zhao, Xiaojuan Qi, Xiaoyong Shen, Jianping Shi, and Jiaya Jia, "Icnet for real-time semantic segmentation on high-resolution images," in $E C C V$, 2018.

[19] Changqian Yu, Jingbo Wang, Chao Peng, Changxin Gao, Gang Yu, and Nong Sang, "Bisenet: Bilateral segmentation network for real-time semantic segmentation," in ECCV, 2018. 